\begin{tabular}{cc} 
Sharif University of Technology \\
Scientia Iranica \\
SCIENTIA & Transactions F: Nanotechnology \\
I RAN I CA & http://scientiairanica.sharif.edu \\
\hline
\end{tabular}

\title{
Investigation into thermophoresis and Brownian motion effects of nanoparticles on radiative heat transfer in Hiemenz flow using spectral method
}

\author{
M.S. Iqbal ${ }^{a}$, A. Ghaffari ${ }^{b, *}$, and I. Mustafa ${ }^{c}$ \\ a. Department of Mathematics Islamabad College for Boys G-6/3, Islamabad 44000, Pakistan. \\ b. Department of Mathematics, University of Education, Lahore (Attock Campus 43600), Pakistan. \\ c. Department of Mathematics, Allama Iqbal Open University, H-8, Islamabad 44000, Pakistan.
}

Received 2 December 2018; received in revised form 13 March 2019; accepted 13 July 2019

\section{KEYWORDS}

Buongiorno nanofluid model;

Hiemenz flow;

Non-linear stretching;

Spectral method.

\begin{abstract}
This study presents a theoretical investigation into thermophoresis and Brownian motion effects on radiative heat transfer in the neighborhood of stagnation point. Thermophoresis and Brownian motion play an important role in thermal and mass concentration analyses, which help to comprehend the major ideas in the disciplines of science and technology. An electrically conducting nanofluid was described by the Buongiorno transport model. The power-law form of the stretching wall velocity made the similarity solution possible. The transformed system of the ordinary differential equations was computed numerically with the efficient and rapid convergent spectral scheme. The obtained results for velocity, temperature, concentration, shear strain, and mass and heat transfer rates were perused for various values of the pertinent parameters. The outcomes divulged that with increase in power-law exponent, mass and heat transfer rates were enhanced. The information on volume and high-temperature transfer rate is provided in tables in this paper. The obtained results well matched the existing results.
\end{abstract}

(C) 2019 Sharif University of Technology. All rights reserved.

\section{Introduction}

In recent years, the need for the development of technology has underscored the importance of fluid flow processes, especially those that include the interaction of numerous phenomena. Convective flow and stretching sheet are a significant part of several engineering applications, e.g., wire drawing, extrusion of polymer sheets from dye, and ground water flows in petroleum industries.

Stagnation point exists in the flows in which the fluid impinges on the solid surface. The stagnation

*. Corresponding author. Tel.: +923325505532

E-mail address: abuzar.ghaffari@ue.edu.pk (A. Ghaffari)

doi: 10.24200 /sci. 2019.52384 .2683 point region possesses the highest pressure, mass, and heat transfer rates. Hiemenz [1], for the first time, considered stagnation-point flow and obtained non-linear ordinary differential equations from NaiverStokes equations by using similarity transformation. Homann [2] investigated an axisymmetric case of stagnation-point flow and Howarth [3] analyzed the same problem numerically. Afterwards, several researchers explored various features of convective heat transfer and stagnation-point flow problems [4-10]. Pop et al. [11] considered the stagnation-point flow over a plate by applying thermal radiation and observed that the boundary layer region was enhanced under the influence of radiation effect. Hayat et al. [12] inspected the stagnation-point flow of a micropolar fluid along a stretching plate. Shateyi and Makinde [13] examined the stagnation-point flow over a radially stretching disk 
and showed that the rise in Biot number enhanced the Nusselt number. Miklavcic and Wang [14] found dual solutions by studying the stagnation-point flow. Mahapatra and Gupta [15] discussed the axisymmetric stagnation-point flow over a stretching surface. They argued that inverted boundary layer existed when free stream velocity was less/higher than the stretching velocity. Mahmood [16] analyzed the effects of the heat source and MHD on stagnation-point flow over a permeable stretching plate with variable heat flux and noticed that heat generation reduced the Nusselt number. Chen [17] considered unsteady mixed convective stagnation-point flow along a stretching surface and noticed that near the surface, reverse flow existed in slip. Makinde [18] analyzed the flow over a surface in the neighborhood of a stagnation point in porous media with heat generation and thermal radiation effects. Boutros et al. [19] studied steady boundary layer flow in a porous medium near the stagnation point region. Nazar et al. [20] investigated the stagnation-point flow on a stretching surface. Hayat et al. [21] discussed the impact of thermal radiation and MHD on Maxwell fluid flow over a flat surface near the stagnation point. Mehmood and Ali [22] discussed the three-dimensional stagnation-point flow over a moving surface for secondgrade fluid and found that a uniformly valid analytic solution existed for all the values of the second-grade parameter.

Using nanoparticles having diameters less than $100 \mathrm{~nm}$ is an effective approach to improving the heat transfer phenomenon in fluids. The heat transfer rate of fluid is considerably altered by dispersing of nanoparticles in the cooling/heating fluid. Heat capacity, surface area, and effective thermal conductivity of base fluid also increase remarkably by introducing nanoparticles into the base fluid. Furthermore, due to the collision of nanoparticles and interaction between fluid particles, the convective phenomenon further increases.

Nanofluid is prepared by mixing the nano-size solid particles into the base liquid having low thermal conductivity, e.g. ethylene glycol or water.

Nanotechnology has many applications to manufacturing related industries, e.g., vehicles engine, drug delivery, making nanochips, currency making, cooling of electronics, etc. Boundary layer theory of fluids with nanoparticles has tremendous uses in polymer engineering, metallurgy, heat exchangers in high heat flux application, insulation of heated bodies, thermal energy storage, drying process, and petrochemical industries.

Forced convective nanofluid flow on a stretching surface is a significant feature of flows because of its applications to metallurgy, polymerization, etc. It is also utilized in petrochemical industry, processing of polymers characterization, and structure and properties of polymers. The nanofluid that encounters stagnation- point flow has maximum heat transfer, maximum pressure, and maximum rate of mass decay. Anwar et al. [23] theoretically studied stagnation-point flow with nanofluid along a non-linear stretching surface and found that thermophoresis and Brownian motion effect amplified the temperature profile and boundary layer region. Wakif et al. [24] investigated thermos-magneto hydrodynamic stability of alumina-water nanofluid by incorporating Chon and Coreione's nanofluid model and reported that the diameter of alumina nanoparticles increased the size of convection cells. Wakif et al. [25] discussed the effects of thermal radiation and magnetic field on the unsteady natural convection Couette flow in $\mathrm{Cu}$-water nanofluid and proved that the rate of heat transfer could be enhanced by increasing either the initial volumetric fraction of nanoparticles or the value of the radiation parameter. Wakif et al. [26] investigated electro-thermo-hydrodynamics instability in the presence of Brownian motion and thermophoresis phenomenon, and showed that electro-thermo-stability in nanofluid could be controlled by the critical AC electric Rayleigh number. Wakif et al. [27] considered generalized Buongiorno's model for nanofluid convection in the presence of a transverse magnetic field and found that thermal stability of nanofluid could be achieved through critical thermal Rayleigh number. Wakif et al. [28] performed linear stability analysis of Newtonian nanofluid in the presence of heat source and showed that at low values of heat source, Lewis number and Rayleigh number would ensure thermal stability of nanofluid. Wakif et al. [29] analyzed stability of conducting nanofluid in a Darcy Brickman porous medium and reported that size of convection cells increased by increasing the Darcy number and decreasing the modified magnetic Chandrasekhar number. Boulahia at al. [30] investigated mixed convection flow in a square cavity and proved that heat transfer could be improved by increasing the concentration of nanoparticles and Richardson number. Boulahia et al. [31] studied the heat transfer of $\mathrm{Cu}$-nanoparticles in a wavy wall cavity and reported that heat transfer rate increased by increasing undulation numbers and decreasing wavy surface amplitude. Boulahia et al. [32] analyzed the free and $\mathrm{MC}$ heat transfers of nanofluid in a square cavity with circular heating and cooling of the cylinder, and showed that heat transfer rate was enhanced by increasing the size of the circular heating body and Rayleigh number.

Tayebi et al. [33] analyzed free convection heat transfer in an annulus between confocal elliptic cylinders filled with CNT-water nanofluid and found that the average Nusselt number was an increasing function of modified Rayleigh numbers. Abbasi and Ghayeni [34] studied saturated air water freezing in two-dimensional stagnation flow on a flat plate. According to their study, below the start temperature of 
freezing, $10^{\circ} \mathrm{C}$ drop in substrate temperature causes a large increase in the ultimate thickness of the ice at farfield air temperature of $5^{\circ} \mathrm{C}$, while it slightly increases the ultimate thickness of the ice at the air temperature of $20^{\circ} \mathrm{C}$. Rana et al. [35] considered hydrothermal characteristics of nanofluid using KKL model with Brownian motion and recommended nano-fluids as a better coolant than the base fluid. Ramly et al. [36] investigated active and passive control of nanoparticles for heat transfer over a stretching sheet under the effect of thermic radiation. They stated that in the case of zero fluxes of nanoparticles, thermophoresis would enhance heat conductivity performance. Karbasi et al. [37] studied the effect of multi-walled carbon nanotubes (MWCNT) on structural and mechanical properties of poly 3-hydroxybutyrate scaffold and stated that low percentage of MWCNT would improve mechanical properties of pure P3HB scaffold. Hakeem et al. [38] numerically investigated the second law for MHD nanofluid flow over the stretching surface and showed that nanoparticles generated more entropy than non-metallic particles. Wang and Dai [39] considered natural convection of nanofluid in an inclined square cavity with non-uniformly heated walls and found that at a fixed Rayleigh number, average Nusselt number was higher for copper nanoparticles. Kasmani et al. [40] reported the effects of thermal radiation, Soret, and Dufour on convective heat transfer of nanoliquid over a wedge in the presence of wall suction and proved that temperature distribution enhanced by raising the values of wedge angle, radiation, Brownian motion, and thermophoresis parameters. Ganji et al. [41] examined magnetite ethylene glycol nanofluid EHD forced convection flow in a lid-driven cavity in the presence of thermal radiation and proved that Nusselt number increased with the rise of Darcy number.

Ibrahim et al. [42] studied convective heat transfer features of MHD boundary layer stagnation-point flow on a stretching surface in nanofluid and showed that Brownian motion and thermophoresis enhanced the temperature profile. Hamad and Ferdows [43] applied group-theoretical methods to investigating the stagnation-point flow over a stretching sheet with heat generation and suction in porous media. They pointed out that the boundary layer flow was smoothened by imposing suction. Alsaedi et al. [44] discussed stagnation-point flow over a surface in nanofluid with Brownian motion and heat generation effects. Khan et al. [45] considered viscous flow on a stretching flat plate in ferrofluid under the influence of viscous dissipation and MHD. They noticed that Nusselt number and the friction factor were larger for kerosene-based ferrofluid than for water-based ferrofluid. Hamad and Pop [46] inspected the impact of heat generation on stagnationpoint nanofluid flow on stretching sheet. Mustafa et al. [47] examined the stagnation-point nanofluid flow over a stretching plate. Ibrahim et al. [48] analyzed MHD effects on nanofluid flow over a stretching sheet. They noted that the thickness of temperature profile increased with a rise in both Brownian motion and thermophoresis parameters. Recently, Hakeem et al. [49] investigated the effects of metallic and non-metallic nano-size particles in the presence of magnetic field and radiation effects on the stretching/shrinking surface. In another study, Hakeem et al. [50] investigated the influence of thermal radiation and non-uniform heat source/sink on the entropy generation rate over a stretching sheet with nanoparticle concentration. They found that entropy generation increased in the case of metallic particles in comparison with non-metallic particles. Notable studies of nanotechnology performed recently can be seen in [51-53].

The objective of this paper is to study the effect of thermal radiation on convective phenomenon in nanofluid over a non-linear stretching surface. The well-known Buongiorno transport model [54,55] for nanofluid is utilized in the development of transport equations. Similarity transformation is used to transform the given ODEs into PDEs. Then, the PDEs are numerically solved by using Chebyshev Spectral method [56-58] scheme. The results of interest, e.g. Nusselt number, Sherwood number, velocity profile, and temperature, will be reported to illustrate the effects of thermophoresis and Brownian motion of nanoparticles as well as thermal radiation on these physical quantities. The feasible branches of the present nanofluid model are micro- and nanoelectromechanical systems, advanced cooling systems, etc., which have applicability to glass fiber production, geothermal reservoirs, manufacturing of plastics, manufacturing processes of continuous casting, metal extrusion, processes involving polymer composites, paper production, textiles, and hot rolling.

\section{Convective transport equations}

We consider a steady, two-dimensional, incompressible, stagnation-point flow impinging on a non-linear continuously stretching horizontal plate. The nonlinear stretching velocity of the flat plate is of the form $\bar{u}_{w}(\bar{x})=u_{0}(\bar{x} / l)^{m}$ [59]. It is also supposed that the moving velocity of the ambient fluid has the form $\bar{u}_{e}(\bar{x})=u_{\infty}(\bar{x} / l)^{m}$ [59], where $u_{\infty}$ and $u_{0}$ are the constants representing dimensions of velocity, $m$ is the power-law velocity exponent, $l$ is the characteristic length, and $u_{0} \lesseqgtr 0$ denotes the direction of motion of the plate. The $x$-axis is taken horizontally and the $y$-axis normal to it. The coordinate system and flow model are displayed in Figure 1.

With the above suppositions, the transport equations for Buongiorno nanofluid model $[47,54,59]$ are written as: 


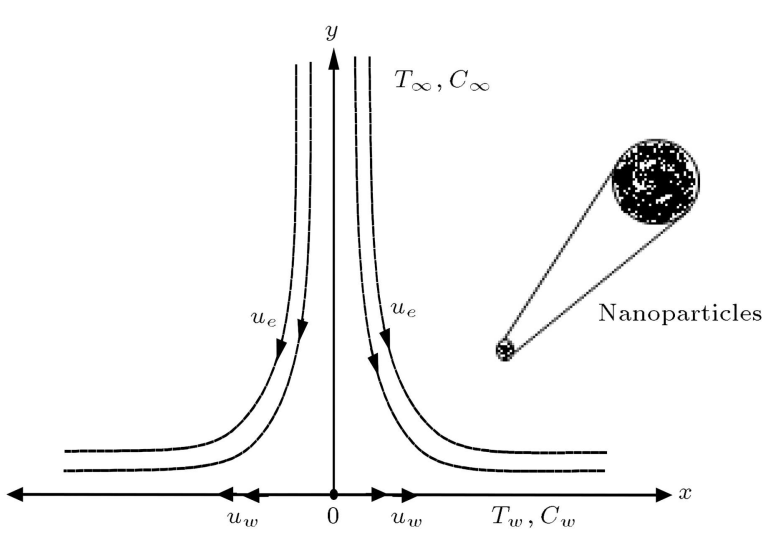

Figure 1. Flow geometry with coordinate system.

Continuity:

$$
\frac{\partial \bar{u}}{\partial \bar{x}}+\frac{\partial \bar{v}}{\partial \bar{y}}=0
$$

Momentum:

$$
\bar{u} \frac{\partial \bar{u}}{\partial \bar{x}}+\bar{v} \frac{\partial \bar{u}}{\partial \bar{y}}=\bar{u}_{e} \frac{d \bar{u}_{e}}{d \bar{x}}+\nu \frac{\partial^{2} \bar{u}}{\partial \bar{y}^{2}},
$$

Energy:

$$
\begin{aligned}
\bar{u} \frac{\partial T}{\partial \bar{x}} & +\bar{v} \frac{\partial T}{\partial \bar{y}}=\alpha \frac{\partial^{2} T}{\partial \bar{y}^{2}}-\frac{1}{\rho c_{p}} \frac{\partial q_{r}}{\partial \bar{y}} \\
& +\tau\left[\frac{D_{T}}{T_{\infty}}\left(\frac{\partial T}{\partial \bar{y}}\right)^{2}+D_{B} \frac{\partial T}{\partial \bar{y}} \frac{\partial C}{\partial \bar{y}}\right],
\end{aligned}
$$

Concentration:

$$
\bar{u} \frac{\partial C}{\partial \bar{x}}+\bar{v} \frac{\partial C}{\partial \bar{y}}=\frac{D_{T}}{T_{\infty}} \frac{\partial^{2} T}{\partial \bar{y}^{2}}+D_{B} \frac{\partial^{2} T}{\partial \bar{y}^{2}},
$$

where $\tau, D_{T}$, and $D_{B}$ are the ratio of heat capacity of nanoparticles to the base fluid, thermophoretic diffusion coefficient, and Brownian diffusion coefficient, respectively, and $(\bar{u}, \bar{v})$ represents velocity components along $(\bar{x}, \bar{y})$ coordinates. Also, $T$ is temperature. The expressions for the Brownian and thermophoresis coefficients are given by $D_{B}=\kappa_{B} T / 3 \pi \mu_{f} d_{p}$ and $D_{T}=\beta \phi \mu_{f} / \rho_{f}$, respectively, where $\kappa_{B}$ is Boltzmann's constant, $d_{p}$ is nanoparticle diameter, $\phi$ is nanoparticle concentration, $\beta$ is expression of proportionality, and $\beta=0.26\left(\kappa_{f} / 2 \kappa_{f}+\kappa_{p}\right)$. For water at room temperature and nanoparticles having the diameters of $1-100 \mathrm{~nm}$, $D_{B} \in 4 \times 10^{-12}$ to $4 \times 10^{-10} \mathrm{~m}^{2} / \mathrm{s}$ (see $[54,60]$ ). The radiative heat flux given in Eq. (3) is approximated by using Rosseland approximation, described as:

$$
q_{r}=-\frac{4 \sigma^{*}}{3 \kappa^{*}} \frac{\partial T^{4}}{\partial y}=-\frac{16 \sigma^{*} T_{\infty}^{3}}{3 \kappa^{*}} \frac{\partial T}{\partial y},
$$

where $\kappa^{*}$ is the mean absorption coefficient and $\sigma^{*}$ is the Stefan-Boltzman constant.
The appropriate boundary conditions [59] following the flow assumptions are:

$$
\begin{aligned}
& \bar{y}=0: \\
& \bar{u}=\bar{u}_{w}(\bar{x}), \quad \bar{v}=0, \quad C=C_{w}, \quad T=T_{w}, \\
& \bar{y} \rightarrow \infty: \\
& \bar{u}=\bar{u}_{e}(\bar{x}), \quad C=C_{\infty}, \quad T=T_{\infty},
\end{aligned}
$$

where $C_{w}$ is plate concentration and $T_{w}$ is plate temperature. Introducing the stream function, $\psi(\eta)$, which satisfies the continuity equation identically, the following similarity variables [59] are presented:

$$
\begin{aligned}
& x=\frac{\bar{x}}{l}, \quad \eta=\operatorname{Re}^{1 / 2} \bar{x}^{\frac{1-m}{2}} \frac{\bar{y}}{l}, \quad u=\frac{\bar{u}}{u_{\infty}}, \\
& v=\frac{\bar{v}}{u_{\infty}} \operatorname{Re}^{1 / 2}, \quad \operatorname{Re}=\frac{u_{\infty} l}{\nu}, \quad \phi(\eta)=\frac{C-C_{\infty}}{C_{w}-C_{\infty}}, \\
& \theta(\eta)=\frac{T-T_{\infty}}{T_{w}-T_{\infty}}, \quad \bar{u}_{e}=\frac{u_{e}}{u_{\infty}}, \quad \psi=x^{\frac{1+m}{2}} f(\eta),
\end{aligned}
$$

which transform systems (2)-(4) to a system of ODEs, described as:

$$
\begin{gathered}
\frac{d^{3} f}{d \eta^{3}}+\left(\frac{m+1}{2}\right) f \frac{d^{2} f}{d \eta^{2}}-m\left(\left(\frac{d f}{d \eta}\right)^{2}-1\right)=0 \\
\frac{1}{\operatorname{Pr}}(1+R d) \frac{d^{2} \theta}{d \eta^{2}}+\left(\frac{m+1}{2}\right) f \frac{d \theta}{d \eta}+N b \frac{d \theta}{d \eta} \frac{d \phi}{d \eta} \\
+N t\left(\frac{d \theta}{d \eta}\right)^{2}=0 \\
\frac{1}{\mathrm{Le}}\left(\frac{d^{2} \phi}{d \eta^{2}}-\frac{N t}{N b}\left(\frac{\operatorname{Pr}}{1+R d}\right)\left(\left(\frac{m+1}{2}\right) f \frac{d \theta}{d \eta}\right.\right. \\
\left.\left.+N b \frac{d \theta}{d \eta} \frac{d \phi}{d \eta}+N t\left(\frac{d \theta}{d \eta}\right)^{2}\right)\right) \\
+\left(\frac{m+1}{2}\right) f \frac{d \phi}{d \eta}=0
\end{gathered}
$$

where $R d=\frac{16 T_{\infty}^{3} \sigma^{*}}{3 \kappa^{*} \kappa}, N b=\frac{\tau D_{B}\left(T_{w}-T_{\infty}\right)}{\nu}, \operatorname{Pr}=\nu / \alpha$, Le $=\alpha / D_{B}$, and $N t=\frac{\tau D_{T}\left(C_{w}-C_{\infty}\right)}{T_{\infty} \nu}$ are the radiation parameter, Brownian motion parameter, Lewis number, thermophoresis parameter, and Prandtl number, respectively. The $N t / N b$ ratio is within the wide range of 0.1 to 1.0 for the typical cases of alumina and copper nanoparticles with the dimension of $10 \mathrm{~nm}$ (see [60]). Also, we have $N t / N b \sim 1 / d_{p}$, where $d_{p}$ is the diameter of the nanoparticle. This indicates that the relation between the theoretical values and 
the experimental results can be controlled in real experiments. Boundary conditions (5) in the nondimensional form are described as:

$$
\begin{aligned}
& f(0)=0, \quad \frac{d f(0)}{d \eta}-V=0, \quad \theta(0)-1=0 \\
& \phi(0)-1=0, \quad \frac{d f(\infty)}{d \eta}-1=0, \quad \theta(\infty)-1=0 \\
& \phi(\infty)-1=0,
\end{aligned}
$$

where $V=\frac{u_{0}}{u_{\infty}}=$ const is the velocity ratio parameter. We can express the outcomes of velocity ratio parameter in 4 different ways; $V>0$ shows that motion of the plate and free stream velocity are in the same direction; $V>1$ and $0<V<1$ correspond to the situations in which the motion of the plate is higher than the free stream velocity and the free stream velocity is faster than the plate, respectively; $V<0$ indicates that the free stream velocity and speed of the plate are in opposite directions; and $V=1$ denotes that the motions of the plate and free stream velocity are equal and hence, in the same direction.

In terms of wall shear stress, $\tau_{w}=\mu(\nabla \bar{u})_{y=0}$, surface heat flux, $q_{w}=-\kappa(\nabla T)_{y=0}$, and surface mass flux, $q_{m}=-D_{B}(\nabla C)_{y=0}$, the coefficient of skin friction, heat transfer rate, and mass transfer rate are respectively written as:

$$
\begin{aligned}
& C_{f}=C_{f x}\left(\operatorname{Re}_{\bar{x}}\right)^{1 / 2}=f^{\prime \prime}(0), \\
& \mathrm{Nu}=\mathrm{Nu}_{x}\left(\operatorname{Re}_{\bar{x}}\right)^{-1 / 2}=-\theta^{\prime}(0), \\
& \mathrm{Sh}=\mathrm{Sh}_{x}\left(\operatorname{Re}_{\bar{x}}\right)^{-1 / 2}=-\phi^{\prime}(0) .
\end{aligned}
$$

Primes numbers are used for derivatives with respect to $\eta$.

\section{Numerical procedure}

Finding a solution to the nonlinear system of differential equations in the theoretical investigation is vital for the entire study. To highly nonlinear complicated differential equations, it is very difficult to find an exact or closed-form solution. In our case, it is quite impossible to find the exact solution. To cope with this situation, we use a numerical technique named Chebyshev Spectral Newton Iterative Scheme (CSNIS). In this scheme, we first use a Newton iterative scheme for the system of nonlinear ODEs in linearized form. In the $(i+1)$ th iterate, for all dependent variables, we write:

$$
\begin{aligned}
& f_{i+1}=f_{i}+\delta f_{i} ; \quad \theta_{i+1}=\theta_{i}+\delta \theta_{i} ; \\
& \phi_{i+1}=\phi_{i}+\delta \phi_{i},
\end{aligned}
$$

where $\delta f_{i}, \delta \theta_{i}$, and $\delta \phi_{i}$ represent minor changes in $f_{i}$, $\theta_{i}$, and $\phi$, respectively. Eqs. (7)-(9) in linearized form can be written as:

$$
\begin{aligned}
a_{1, i} \delta & \left(\frac{d^{3} f}{d \eta^{3}}\right)_{i}+a_{2, i} \delta\left(\frac{d^{2} f}{d \eta^{2}}\right)_{i}+a_{3, i} \delta\left(\frac{d f}{d \eta}\right)_{i} \\
& +a_{4, i} \delta f_{i}=R_{1, i} \\
b_{1, i} \delta & \left(\frac{d^{2} \theta}{d \eta^{2}}\right)_{i}+b_{2, i} \delta\left(\frac{d \theta}{d \eta}\right)_{i}+c_{1, i} \delta\left(\frac{d \phi}{d \eta}\right)_{i} \\
& +a_{5, i} \delta f_{i}=R_{2, i}, \\
c_{2, i} \delta & \left(\frac{d^{2} \phi}{d \eta^{2}}\right)_{i}+c_{3, i} \delta\left(\frac{d \phi}{d \eta}\right)_{i}+b_{3, i} \delta\left(\frac{d \theta}{d \eta}\right)_{i} \\
& +a_{6, i} \delta f_{i}=R_{3, i} .
\end{aligned}
$$

Boundary conditions take the following form:

$$
\begin{aligned}
& \left.\delta f_{i}\right|_{\eta=0}=0-\left.f_{i}\right|_{\eta=0}, \\
& \left.\delta\left(\frac{d f}{d \eta}\right)_{i}\right|_{\eta=0}=V-\left.\left(\frac{d f}{d \eta}\right)_{i}\right|_{\eta=0}, \\
& \left.\delta \theta_{i}\right|_{\eta=0}=1-\left.\theta_{i}\right|_{\eta=0}, \\
& \left.\delta \phi_{i}\right|_{\eta=0}=1-\left.\phi_{i}\right|_{\eta=0}, \\
& \left.\delta\left(\frac{d f}{d \eta}\right)_{i}\right|_{\eta=\infty}=1-\left.\left(\frac{d f}{d \eta}\right)_{i}\right|_{\eta=\infty}, \\
& \left.\delta \theta_{i}\right|_{\eta=\infty}=0-\left.\theta_{i}\right|_{\eta=\infty}, \\
& \left.\delta \phi_{i}\right|_{\eta=\infty}=0-\left.\phi_{i}\right|_{\eta=\infty} .
\end{aligned}
$$

The coefficients $a_{-, i}$ 's, $b_{-, i}$ 's, $c_{-, i}$ 's, and $R_{-, i}$ 's are:

$$
\begin{aligned}
a_{1, i}= & 1, \quad a_{2, i}=\left(\frac{m+1}{2}\right) f_{i}, \\
a_{3, i}= & -2 m\left(\frac{d f}{d \eta}\right)_{i}, \\
a_{4, i}= & \left(\frac{m+1}{2}\right)\left(\frac{d^{2} f}{d \eta^{2}}\right)_{i}, \\
a_{5, i}= & \left(\frac{m+1}{2}\right)\left(\frac{d \theta}{d \eta}\right)_{i}, \\
a_{6, i}= & \left(\frac{m+1}{2}\right)\left(\frac{d \phi}{d \eta}\right)_{i} \\
& -\frac{1}{\operatorname{Le}} \frac{N t}{N b} \frac{\operatorname{Pr}}{1+R d}\left(\frac{m+1}{2}\right)\left(\frac{d \theta}{d \eta}\right)_{i}, \\
b_{1, i}= & \frac{1+R d}{\operatorname{Pr}},
\end{aligned}
$$




$$
\begin{aligned}
& b_{2, i}=\left(\frac{m+1}{2}\right) f_{i},+2 N t\left(\frac{d \theta}{d \eta}\right)_{i}+N b\left(\frac{d \phi}{d \eta}\right)_{i}, \\
& b_{3, i}=-\frac{1}{\operatorname{Le}} \frac{N t}{N b} \frac{\operatorname{Pr}}{1+R d}\left(\frac{m+1}{2}\right) f_{i} \\
& -\frac{N t}{\mathrm{Le}} \frac{\operatorname{Pr}}{1+R d}\left(\frac{d \phi}{d \eta}\right)_{i} \\
& -\frac{2}{\mathrm{Le}} \frac{N t^{2}}{N b} \frac{\operatorname{Pr}}{1+R d}\left(\frac{d \theta}{d \eta}\right)_{i}, \\
& c_{1, i}=N b\left(\frac{d \theta}{d \eta}\right)_{i}, \quad c_{2, i}=\frac{1}{\mathrm{Le}}, \\
& c_{3, i}=N b\left(\frac{d \theta}{d \eta}\right)_{i}+\left(\frac{m+1}{2}\right) f_{i}, \\
& R_{1, i}=-\left(\frac{d^{3} f}{d \eta^{3}}\right)_{i}-\left(\frac{m+1}{2}\right) f \frac{d^{2} f}{d \eta^{2}} \\
& +m\left(\left(\frac{d f}{d \eta}\right)_{i}^{2}-1\right) \\
& R_{2, i}=-\frac{1}{\operatorname{Pr}}(1+R d)\left(\frac{d^{2} \theta}{d \eta^{2}}\right)_{i}-\left(\frac{m+1}{2}\right) f_{i}\left(\frac{d \theta}{d \eta}\right)_{i} \\
& -N b\left(\frac{d \theta}{d \eta}\right)_{i}\left(\frac{d \phi}{d \eta}\right)_{i}+N t\left(\frac{d \theta}{d \eta}\right)_{i}^{2}, \\
& R_{3, i}=-\frac{1}{\mathrm{Le}}\left(\left(\frac{d^{2} \phi}{d \eta^{2}}\right)_{i}-\frac{N t}{N b}\left(\frac{\operatorname{Pr}}{1+R d}\right)\right. \\
& \left(\left(\frac{m+1}{2}\right) f_{i}\left(\frac{d \theta}{d \eta}\right)_{i}+N b\left(\frac{d \theta}{d \eta}\right)_{i}\left(\frac{d \phi}{d \eta}\right)_{i}\right. \\
& \left.\left.+N t\left(\frac{d \theta}{d \eta}\right)_{i}^{2}\right)\right)-\left(\frac{m+1}{2}\right) f_{i}\left(\frac{d \phi}{d \eta}\right)_{i} .
\end{aligned}
$$

The linearized system in Eqs. (13) with boundary conditions (14) is solved using the Chebyshev Spectral Collocation method [33-35]. The physical domain from 0 to $\infty$ is first converted into the finite domain of 0 to $L$. The value of $L$ is chosen sufficiently large. This finite domain is then transformed into -1 to 1 by using relation $\xi=\frac{2 \eta}{L}-1$. The Gauss-Lobatto node points are used form -1 to 1 , which are calculated by the relation $\xi j=\cos (\pi j / N), j=0,1,2, \cdots N$. The differential matrix $D$ is commonly known as Chebyshev differentiation matrix. Applying collocation method to Eqs. (13) and (14), the following matrix is obtained:

$$
\left[\begin{array}{lll}
A_{11} & A_{12} & A_{13} \\
A_{21} & A_{22} & A_{23} \\
A_{31} & A_{32} & A_{33}
\end{array}\right]\left[\begin{array}{l}
\delta f_{i} \\
\delta \theta_{i} \\
\delta \phi_{i}
\end{array}\right]=\left[\begin{array}{l}
R_{1, i} \\
R_{2, i} \\
R_{3, i}
\end{array}\right],
$$

where:

$$
\begin{aligned}
& A_{11}=a_{1, i} D^{3}+a_{2, i} D^{2}+a_{3, i} D+a_{4, i} I, \\
& A_{12}=\mathbf{0}, \quad A_{13}=\mathbf{0}, \\
& A_{21}=a_{5, i} I, \quad A_{22}=b_{1, i} D^{2}+b_{2, i} D, \\
& A_{23}=c_{1, i} D, \\
& A_{31}=a_{6, i} I, \quad A_{32}=b_{3, i} D, \\
& A_{33}=c_{2, i} D^{2}+c_{3, i} D .
\end{aligned}
$$

$I$ is the identity matrix and $a_{-, i}$ 's, $b_{-, i}$ 's, $c_{-, i}$ 's, and $R_{-, i}$ 's as given in Eq. (15). The unknown $\delta f_{i}, \delta \theta_{i}$, and $\delta \phi_{i}$ are calculated by the linearized system given in Eq. (16). After every iteration, the functions $f_{i+1}=$ $f_{i}+\delta f_{i}, \theta_{i+1}=\theta_{i}+\delta \theta$, and $\phi_{i+1}=\phi_{i}+\delta \phi_{i}$ are updated to a more refined form. Finally, after a few iterations, we obtain our required results.

\section{Validation of results}

The associated boundary conditions and transformed system of Eqs. (7)-(9) and (10) are solved by using CSNIS [56-58]. In this study, we utilize various ranges of $N b, N t$, and $m$, and fixed values of Pr, Le, and $R d$. The numerical procedure is coded in Matlab software. $\eta_{\max }=15$ is proven enough to attain the boundary conditions for all parameters, asymptotically. To examine validity of the results, a comparison with the published data in the literature is made. The present results in the form of skin friction obtained by CSNIS for various values of power-law index $m$ are compared with Yih [61], Uddin et al. [59], and Cebeci and Bradshaw [62] in Table 1. The table illustrates the accuracy and validity of the current numerical procedure. The outcomes are displayed in tables and figures, and discussed in the following section.

\section{Results and discussion}

Graphical representations of velocity, concentration of nanoparticles, and temperature are given for different pertinent parameters, such as $m, N b$, and $N t$ with fixed values of Le, $\mathrm{Pr}$, and $R d$ in Figures 2-10. Table 2 represents $\mathrm{Nu}$ and $\mathrm{Sh}$ against different values of $N t$, $N b$, and $V$ with $\operatorname{Pr}=7.0, \mathrm{Le}=10, m=2.0$, and $R d=0.1$.

Figure 2 depicts the influence of $m$ on velocity distribution for various values of velocity ratio $V$. The figure illustrates that the velocity increases when the plate is stationary, velocity profile remains unchanged when free steam velocity and movement speed of the plate are equal, and velocity decreases when speed of 
Table 1. Comparison of the values of $f^{\prime \prime}(0)$ with $V=0$.

\begin{tabular}{ccccc}
\hline \multirow{2}{*}{} & \multicolumn{4}{c}{$\boldsymbol{f}^{\prime \prime}(\mathbf{0})$} \\
\cline { 2 - 5 } & Present study (CSNIS) & Cebeci and Bradshaw [62] & Uddin et al. [59] & Yih [61] \\
\hline-0.05 & 0.21382164 & 0.2134838 & 0.21351 & 0.213484 \\
0.0 & 0.33205766 & 0.3320574 & 0.33206 & 0.332057 \\
$1 / 3$ & 0.75744844 & 0.7574476 & 0.75745 & 0.757448 \\
1 & 1.23259013 & 1.2325876 & 1.23259 & 1.232588 \\
\hline
\end{tabular}

Table 2. Numerical data for $\mathrm{Nu}$ and $\mathrm{Sh}$ with variations of $N t$ and $N b$ and $\operatorname{Pr}=7.0, \mathrm{Le}=10, R d=0.1$, and $m=2.0$.

\begin{tabular}{ccccccc}
\hline \multirow{2}{*}{$\boldsymbol{N} \boldsymbol{t} \mathbf{N} \boldsymbol{b}$} & \multicolumn{7}{c}{$\boldsymbol{N} \boldsymbol{b}=\mathbf{0 . 1} \mathbf{0}$} \\
\cline { 2 - 7 } & $\mathbf{N u}$ & $\mathbf{S h}$ & $\mathbf{N u}$ & $\mathbf{S h}$ & $\mathbf{N u}$ & $\mathbf{S h}$ \\
\hline $\mathbf{0 . 1}$ & 0.76295 & 1.7652 & 0.46752 & 2.3976 & 0.30438 & 3.1446 \\
$\mathbf{0 . 3}$ & 0.32290 & 1.8180 & 0.19474 & 2.0826 & 0.12606 & 2.3121 \\
$\mathbf{0 . 5}$ & 0.12297 & 1.7878 & 0.07393 & 1.9486 & 0.04778 & 2.0743 \\
\hline & & \multicolumn{7}{c}{$\boldsymbol{V}=\mathbf{1}$} \\
\hline $\mathbf{0 . 1}$ & 1.3163 & 2.8960 & 0.83532 & 3.3950 \\
$\mathbf{0 . 3}$ & 0.54804 & 3.2104 & 0.34307 & 3.5131 & 0.56136 & 4.2355 \\
$\mathbf{0 . 5}$ & 0.2064 & 3.2007 & 0.12853 & 3.4024 & 0.085603 & 3.5656 \\
\hline & & \multicolumn{7}{c}{$\boldsymbol{V}=\mathbf{2}$} \\
\hline $\mathbf{0 . 1}$ & 1.7364 & 3.7629 & 1.1143 & 4.1960 & 0.75785 & 5.0966 \\
$\mathbf{0 . 3}$ & 0.72064 & 4.2465 & 0.45681 & 4.5806 & 0.30890 & 4.8991 \\
$\mathbf{0 . 5}$ & 0.27058 & 4.2475 & 0.17076 & 4.4781 & 0.11523 & 4.6632 \\
\hline
\end{tabular}

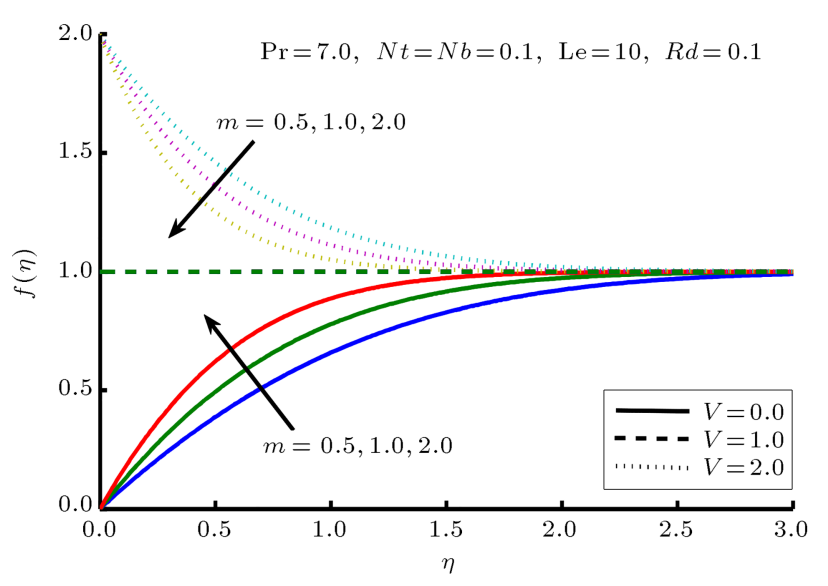

Figure 2. Velocity distribution for different values of $m$.

the plate is higher than the free stream velocity. Variation of $N t$ with temperature distribution is displayed in Figure 3. Temperature distribution is enhanced by increasing $N t$. This is due to the fact that increase in $N t$ enhances temperature within the boundary layer and, consequently, increases thermal layer thickness. The effect of $\mathrm{Nb}$ on nanoparticle concentration distribution is plotted in Figure 4. Increase in $N b$ results in a decrease in the concentration profile. Physically, Sherwood number increases with an increase in $\mathrm{Nb}$, leading to a reduction in the concentration profile.

Variation of $\mathrm{Nu}$ for various values of $N t, N b$, and

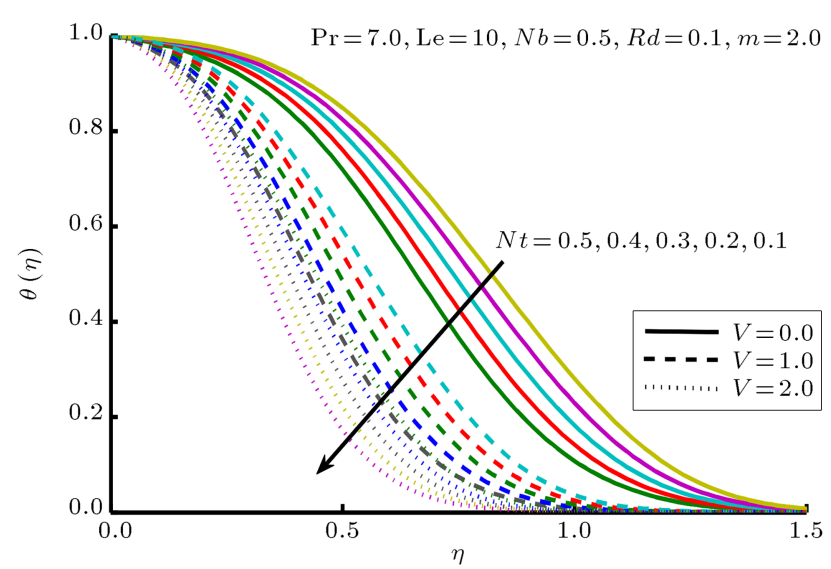

Figure 3. Impact of $N t$ on temperature distribution.

$V$ is presented in Figure 5. It is observed that $\mathrm{Nu}$ is reduced as $N b$ increases, and this decrease becomes linear as $N t$ increases. Furthermore, $\mathrm{Nu}$ is maximum when the plate is moving with a speed higher than the free stream velocity and the increase in $\mathrm{Nu}$ is minimum when the plate is stationary. Also, when plate speed is equal to the free stream velocity, $\mathrm{Nu}$ lies between maximum and minimum levels, as shown in Figure 5. The influence of change in $N b$ and $N t$ at different values of velocity ratio parameter $V$ on $\mathrm{Sh}$ is depicted in Figure 6. It is noticed that the increase in $\mathrm{Sh}$ is maximum when the plate is moving with a speed higher 


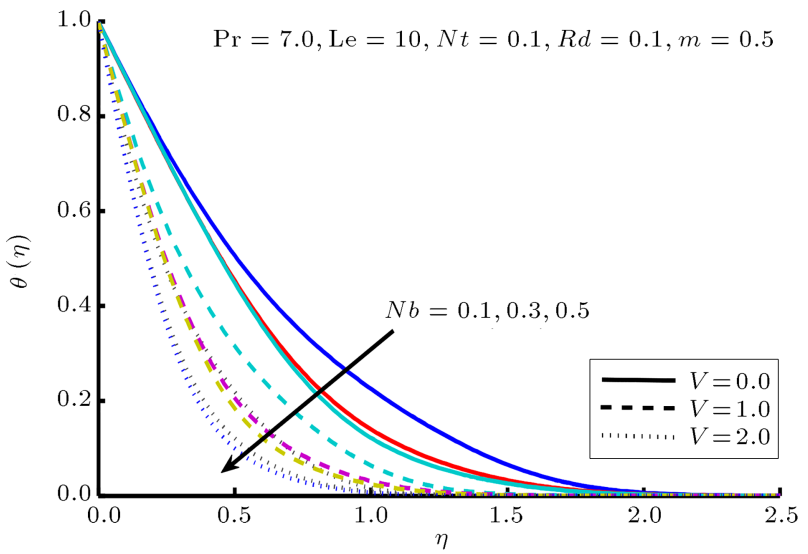

Figure 4. Impact of $N b$ on concentration profile.

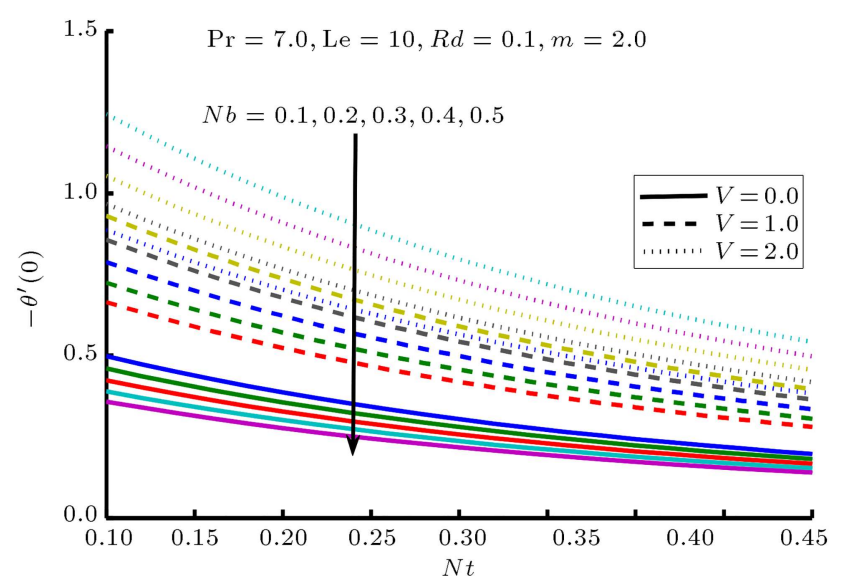

Figure 5. Variations of Nusselt number versus $N t$ for different values of $N b$.

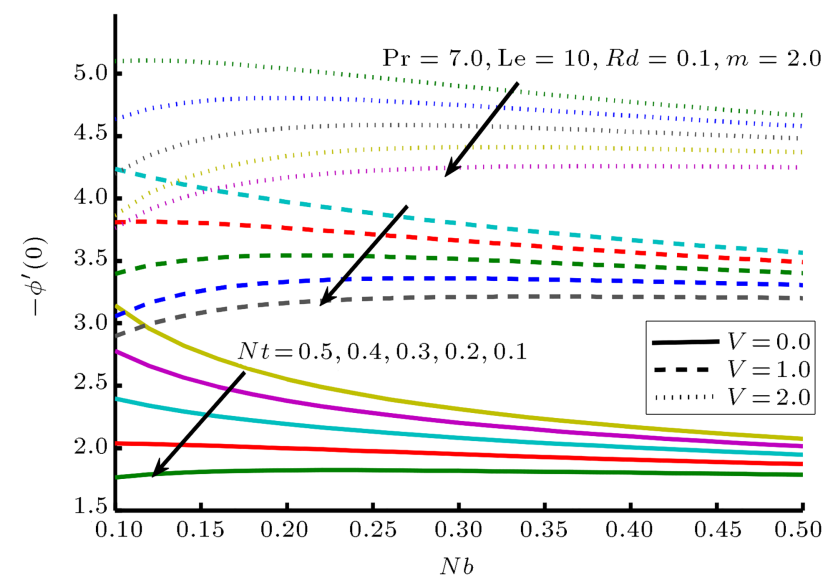

Figure 6. Impact of $N t$ and $N b$ on Sherwood.

than the free stream velocity and the increase in Sh is minimum when the plate is stationary. In addition, moderate increase in Sh is observed when plate speed is equal to the free stream velocity. Figure 7 depicts the influence of $N t$ and $m$ on $\mathrm{Nu}$. It is shown that $\mathrm{Nu}$ is reduced with an increase in $N t$ and enhanced with increase in $m$. Figure 8 illustrates the variation in $\mathrm{Sh}$

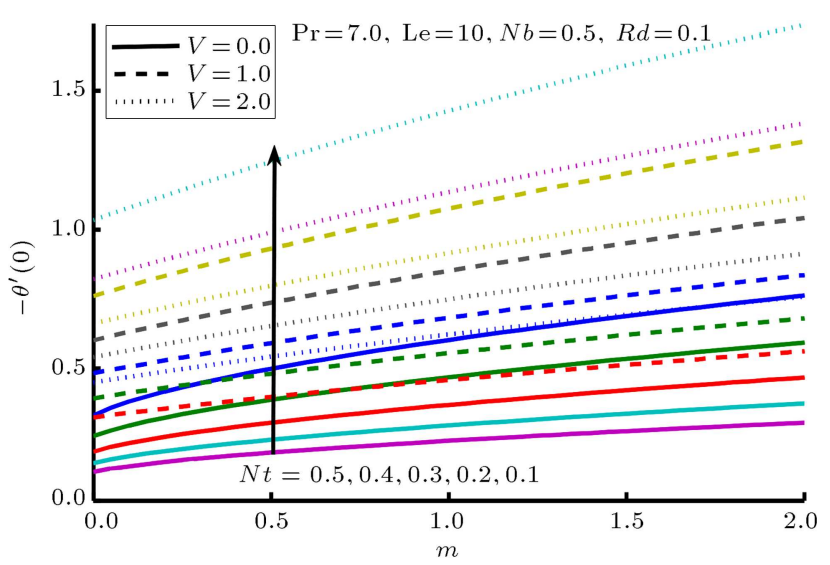

Figure 7. Impact of $N t$ and $m$ on Nusselt number.

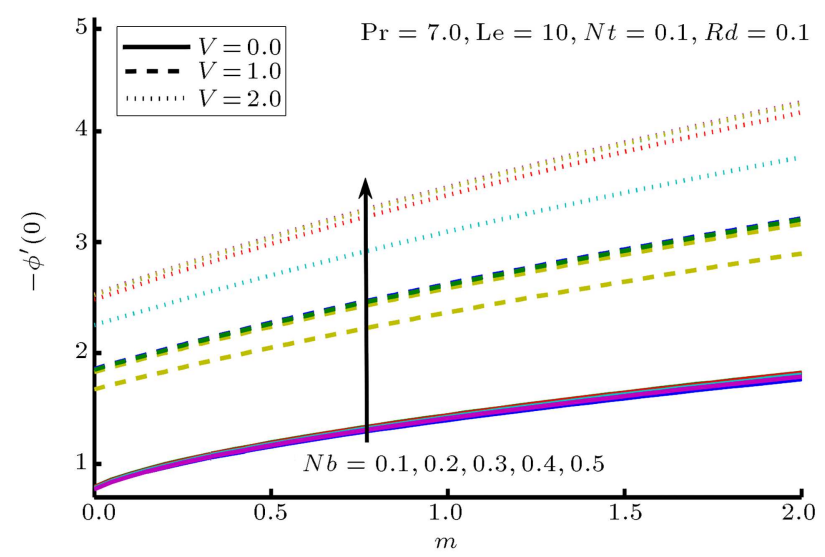

Figure 8. Variation of Sherwood number with $N b$ and $m$.

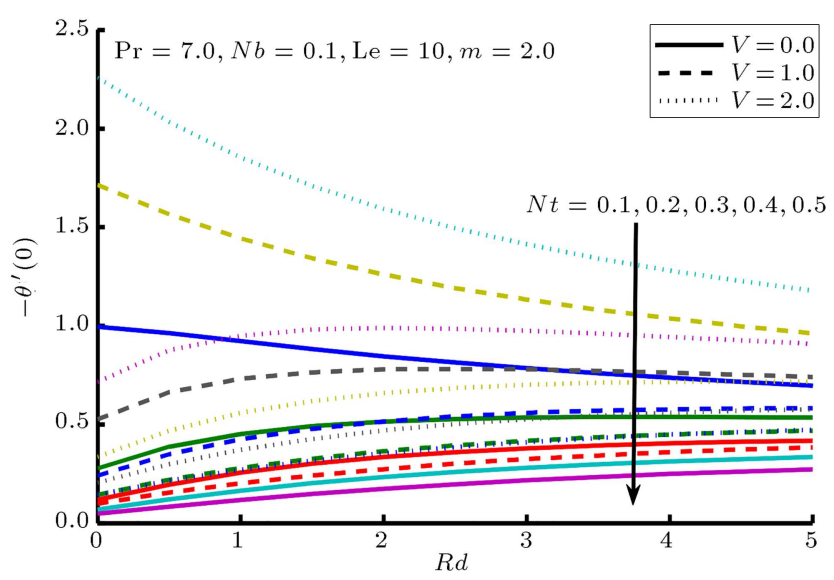

Figure 9. Impact of $N t$ on $\mathrm{Nu}$ and $R d$.

by varying $N b$ and $m$. The figure reveals that $\mathrm{Sh}$ rises with the increase in $N b$. Furthermore, an increasing trend is observed in Sh as $m$ increases. In both plots, enhancement of $\mathrm{Nu}$ and $\mathrm{Sh}$ is observed when plate speed is faster than free stream velocity. Also, increase in both values is minimum when the plate is stationary and moderate when plate speed is equal to free stream velocity. Figures 9 and 10 display the effects of $N t, N b$, and $R d$ on $\mathrm{Nu}$ and $\mathrm{Sh}$. It is shown that $\mathrm{Nu}$ decreases 


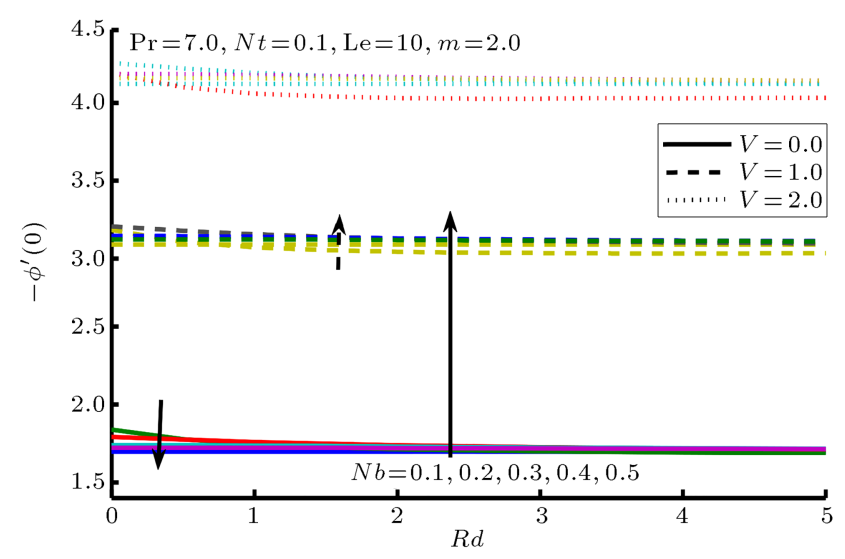

Figure 10. Variation of Sh against $R d$ with various values of $N b$.

with increase in $N t$ and this decrease becomes linear as radiation parameter is reinforced. On the other hand, Sh increases with increase in $N b$, but this increase is minor and becomes linear as radiation parameter is enhanced. The effects of various values of velocity ratio parameter are similar to those discussed in Figures 5-8.

Numerical data for $\mathrm{Nu}$ and $\mathrm{Sh}$ with various values of $N t$ and $N b$ at three different positions of the plate are presented in Table 2 for fixed values of the $\operatorname{Pr}, R d$, Le, and $m$. Nu decreases with increase in both $N t$ and $N b$, but this decrease is faster with increase in $N b$ than with increase in $N t$. Sh rises with increase in $N t$ and $\mathrm{Nb}$, although the rate of increase is slightly attenuated at higher values of $\mathrm{Nb}(\mathrm{Nb}=0.5)$. Table 2 shows that numerical values of $\mathrm{Nu}$ and $\mathrm{Sh}$ are higher when the plate is moving with a speed higher than the free stream velocity and lower when the plate is stationary.

\section{Concluding remarks}

In this study, a theoretical nonlinear Buongiorno nanofluid model for steady-state two-dimensional flow on a radiating horizontal stretching plate was examined. The Chebyshev spectral method was implemented to numerically evaluate the boundary value problem. The numerical results were compared with the results of earlier studies for regular fluids. The outcomes of the present study in brief are the following:

- Reduction in Nusselt number and enhancement of Sherwood number were seen with increase in Brownian motion and thermophoresis parameters;

- Thermal radiation reduced Nusselt number and enhanced Sherwood number;

- Power law index enhanced Sherwood number as well as Nusselt number.

\section{Nomenclature}

C Solutal concentration
$C_{\infty} \quad$ Ambient solutal concentration

$C_{f} \quad$ Skin friction coefficient

$C_{w} \quad$ Solutal concentration at the wall

$D_{B} \quad$ Brownian diffusion coefficient

$D_{T} \quad$ Thermophoretic diffusion coefficient

$f \quad$ Dimensionless normal component of flow

$M \quad$ Dimensionless magnetic parameter

$\mathrm{Nb} \quad$ Brownian motion parameter

Nt Thermophoresis parameter

$\mathrm{Nu} \quad$ Nusselt number

Pr Prandtl number

$\operatorname{Re}_{x} \quad$ Local Reynolds number

Sc Schmidt number

$\mathrm{Sh}_{x} \quad$ Local nanoparticle Sherwood number

$T \quad$ Temperature of the fluid

$T_{\infty} \quad$ Ambient fluid temperature

$T_{w} \quad$ Surface temperature

$\bar{u}, \bar{v} \quad$ Dimensional velocity components in $\bar{x}$ and $\bar{y}$ directions

$u, v \quad$ Dimensionless velocity components in $x$ and $y$ directions

$U_{e} \quad$ Free stream velocity

$U_{w} \quad$ Velocity at the wall

$\bar{x}, \bar{y} \quad$ Coordinates along and normal to the surface in dimensional form

$x, y \quad$ Coordinates along and normal to the surface in dimensionless form

\section{Greek symbols}

$\theta \quad$ Dimensionless temperature

$\phi \quad$ Dimensionless concentration

$\tau_{w} \quad$ Wall shear stress

$\psi \quad$ Stream function

$\nu \quad$ Kinematic viscosity

$\mu \quad$ Dynamic viscosity

$\rho \quad$ Fluid density

$\lambda_{1} \quad$ Relaxation time of the material

$(\rho C)_{f} \quad$ Heat capacity of the fluid

$(\rho C)_{p} \quad$ Effective heat capacity of the nanoparticle material

\section{References}

1. Hiemenz, K. "Die Grenzschicht an einem in den Gleichformingen Flussigkeitsstrom eingetauchten Graden Kreiszylinder", Dinglers Polytech. J., 326, pp. 321-324 (1911).

2. Homann, F. "Der Einfluss grosser Zahigkeit bei der Stromung um den Zylinder und um die Kugel", $Z$. Angew Math. Mech., 16, pp. 153-164 (1936). 
3. Howarth, L. "On the solution of the laminar boundary layer equations", Proc. R. Soc. A, 164(919), pp. 547579 (1938).

4. Chiam, T.C. "Stagnation-point flow towards a stretching plate", J. Phys. Soc. Jpn, 63(6), pp. 2443-2444 (1994).

5. Layek, G.C., Mukhopadhyay, S., and Samad, S.A. "Heat and mass transfer analysis for boundary layer stagnation point flow towards a heated porous stretching sheet with heat absorption/generation and suction/blowing", Int. Commun. Heat Mass Transfer, 34(3), pp. 347-356 (2007).

6. Ishak, A., Nazar, R., and Pop, I. "Mixed convection stagnation point flow of a micropolar fluid towards a stretching sheet", Meccanica, 43(4), pp. 411-418 (2008).

7. Bhattacharyya, K., Mukhopadhyay, S., and Layek, G.C. "Reactive solute transfer in magneto hydrodynamic boundary layer stagnation-point flow over a stretching sheet with suction/ blowing", Chem. Eng. Commun., 199(3), pp. 368-383 (2012).

8. Mabood, F., Khan, W.A., and Ismail, A.M. "MHD stagnation point flow and heat transfer impinging on stretching sheet with chemical reaction and transpiration”, Chem. Eng., 273, pp. 430-437 (2015).

9. Hayat, T., Anwar, M.S., Farooq, M., and Alsaedi, A. "MHD stagnation point flow of second grade fluid over a stretching cylinder with heat and mass transfer", Int. J. Non. Sci. Num. Sim., 15(6), pp. 365-376 (2014).

10. Rashidi, M.M. and Erfani, E. "A new analytical study of MHD stagnation-point flow in porous media with heat transfer", Comp. Fluids, 40(1), pp. 172-178 (2011).

11. Pop, S.R., Grosan, T., and Pop, I. "Radiation effects on the flow near the stagnation point of a stretching sheet", Technische Mechanik, 25(2), pp. 100-106 (2004).

12. Hayat, T., Javed, T., and Abbas, Z. "MHD flow of a micropolar fluid near a stagnation point towards a non-linear stretching surface", Non-Linear Anal. Real World Appl., 10, pp. 1514-1526 (2009).

13. Shateyi, S. and Makinde, O.D. "Hydromagnetic stagnation-point flow towards a radially stretching convectively heated disk", Math. Prob. Eng., 2013, ID 616947 (2013).

14. Miklavcic, M. and Wang, C.Y. "Viscous flow due to a shrinking sheet", Appl. Math., 64, pp. 283-290 (2006).

15. Mahapatra, T.R. and Gupta, A.G. "Heat transfer in stagnation point flow towards a stretching sheet", Heat Mass Transfer, 38, pp. 517-521 (2002).

16. Mahmoud, M.A. "Hydromagnetic stagnation point flow towards a porous stretching sheet with variable surface heat flux in the presence of heat generation", Chem. Eng. Commun., 198(7), pp. 837-846 (2011).
17. Chen, H. "Mixed convection unsteady stagnation point flow towards a stretching sheet with slip effects and noticed that the reverse flow occurs near the sheet", Math. Prob. Eng., 2014, ID 435697 (2014).

18. Makinde, O.D. "Heat and mass transfer by MHD mixed convection stagnation point flow toward a vertical plate embedded in a highly porous medium with radiation and internal heat generation", Meccanica, 47(5), pp. 1173-1184 (2012).

19. Boutros, Y.Z., Abd-el-Malek, M.B., Badran, N.A., and Hassan, H.S. "Lie-group method of solution for steady two-dimensional boundary-layer stagnation-point flow towards a heated stretching sheet placed in a porous medium", Meccanica, 41, pp. 681-691 (2006).

20. Nazar, R., Amin, N., Filip, D., and Pop, I. "Stagnation point flow of a micropolar fluid towards a stretching sheet", Int. J. Non-Linear Mech., 39, pp. 1227-1235 (2004).

21. Hayat, T., Waqas, M., Shehzad, S.A., and Alsaedi, A. "Mixed convection radiative flow of Maxwell fluid near stagnation point with convective condition", J. Mech., 29, pp. 403-409 (2013).

22. Mehmood, A. and Ali, A. "An explicit analytic solution of steady three-dimensional stagnation point flow of second grade fluid toward a heated plate", J. App. Mech. Trans. ASME., 75(6), p. 061003 (2008).

23. Anwar, M.I., Khan, I., Hussnan, A., Salleh, M.Z., and Sharidan, S. "Stagnation point flow of a nanofluid over a nonlinear stretching sheet", W. App. Sci. J., 23(8), pp. 998-1006 (2013).

24. Wakif, A., Boulahia, Z., Animasaun, I.L., Afridi, M.I., Qasimd, M., and Sehaqui, R. "Magneto- convection of alumina-water nanofluid within thin horizontal layers using the revised generalized Buonjiorno's model", Frontiers Heat Mass Transfer, 12(3), pp. 1-15 (2019).

25. Wakif, A., Boulahia, Z., Ali, F., Eid, M.R., and Sehaqui, R. "Numerical analysis of the unsteady natural convection MHD couette nanofluid flow in the presence of thermal radiation using single and twophase nanofluid models for Cu-water nanofluids", Int. J. Appl. Comp. Math., 4(3), p. 81 (2018).

26. Wakif, A., Boulahia, Z., and Sehaqui, R. "A semianalytical analysis of electro-thermo-hydrodynamic stability in dielectric nanofluids using Buongiorno's mathematical model together with more realistic boundary conditions", Results Phys., 9, pp. 1438-1454 (2018).

27. Wakif, A., Boulahia, Z., Mishra, S.R., Rashidi, M.M., and Sehaqui, R. "Influence of a uniform transverse magnetic field on the thermo-hydrodynamic stability in water-based nanofluids with metallic nanoparticles using the generalized Buongiorno's mathematical model", Eur. Phys. J. Plus, 133(5), pp. 181 (2018).

28. Wakif, A., Boulahia, Z., and Sehaqui, R. "Numerical study of the onset of convection in a Newtonian nanofluid layer with spatially uniform and non-uniform internal heating", J. Nanofluids, 6(1), pp. 136-148 (2017). 
29. Wakif, A., Boulahia, Z., and Sehaqui, R. "Numerical analysis of the onset of longitudinal convective rolls in a porous medium saturated by an electrically conducting nanofluid in the presence of an external magnetic field", Results Phys., 7, pp. 2134-2152 (2017).

30. Boulahia, Z., Wakif, A., and Sehaqui, R. "Heat transfer and $\mathrm{Cu}$-water nanofluid flow in a ventilated cavity having central cooling cylinder and heated from the below considering three different outlet port locations", Frontiers Heat Mass Transfer, 11, p. 11 (2018).

31. Boulahia, Z., Wakif, A., and Sehaqui, R. "Modeling of free convection heat transfer utilizing nanofluid inside a wavy enclosure with a pair of hot and cold cylinders", Frontiers Heat Mass Transfer, 8(14), pp. 1-10 (2017).

32. Boulahia, Z., Wakif, A., Chamkha, A.J., and Sehaqui, R. "Numerical study of natural and mixed convection in a square cavity filled by a $\mathrm{Cu}$-water nanofluid with circular heating and cooling cylinders", Mech. Ind., 18(5), p. $502(2017)$.

33. Tayebi, T., Chamkha, A.J., and Djezzar, M. "Natural convection of CNT-water nanofluid in an annular space between confocal elliptic cylinders with constant heat flux on inner wall", Sci. Iran, 26(5), pp. 2770-2783 (2018).

34. Abbasi, A.S. and Ghayen, M. "Vapor solidification of saturated air in two-dimensional stagnation flow", Sci. Iran (2018) DOI: 10.24200/SCI.2018.4956.1007.

35. Rana, S., Nawaza, M., and Qureshi, I.H. "Numerical study of hydrothermal characteristics in nanofluid using KKL model with Brownian motion", Sci. Iran, 26(3), pp. 1931-1943 (2019).

36. Ramly, N.A., Sivasankaran, S., and Noor, N.F.F. "Zero and nonzero normal fluxes of thermal radiative boundary layer flow of nanofluid over a radially stretched surface", Sci. Iran, 24(6), pp. 2895-2903 (2017).

37. Karbasi, S., Zarei, M., and Foroughi, M.R. "Effects of Multi-Wall carbon Nano-Tubes (MWNTs) on structural and mechanical properties of electrospun poly (3hydroxybutyrate) scaffold for tissue engineering applications", Sci. Iran, Transactions F, Nanotechnology, 23(6), p. 3145 (2016).

38. Hakeem, A.A., Govindaraju, M., Ganga, B., and Kayalvizhi, M. "Second law analysis for radiative MHD slip flow of a nanofluid over a stretching sheet with nonuniform heat source effect", Sci. Iran, Transactions F, Nanotechnology, 23(3), p. 1524 (2016).

39. Wanga, X. and Dai, W. "Numerical investigation on natural convection of nanofluids in an inclined square enclosure with non-uniform heated walls", Sci. Iran, 26(4), pp. 2311-2328 (2019).
40. Kasmani, R.M., Sivasankaran, S., Bhuvaneswari, M., Alshomrani, A.S., and Siri, Z. "Soret and Dufour effects on doubly diffusive convection of nanofluid over a wedge in the presence of thermal radiation and suction", Sci. Iran, 26(5), pp. 2817-2826 (2019).

41. Ganji, D.D., Sheikholeslami, M., Zhixiong, L., and Hosseinnejad, R. "Numerical simulation of thermal radiative heat transfer effects on $\mathrm{Fe}_{3} \mathrm{O}_{4}$-Ethylene glycol nanofluid EHD flow in a porous enclosure", Sci. Iran, 26(3), pp. 1405-1414 (2019).

42. Ibrahim, W., Shankar, B., Mahantesh, M., and Nandeppanavar, M. "MHD stagnation point flow and heat transfer due to nanofluid towards a stretching sheet", Int. J. Heat Mass Transfer, 56, pp. 1-9 (2013).

43. Hamad, M.A.A. and Ferdows, M. "Similarity solution of boundary layer stagnation-point flow towards a heated porous stretching sheet saturated with a nanofluid with heat absorption/generation and injection/blowing: a lie group analysis", Comp. Nonlinear Sci. and Num. Simul., 17, pp. 132-140 (2011).

44. Alsaedi, A., Awais, M., and Hayat, T. "Effects of heat generation/absorption on stagnation-point flow of nanofluid over a surface with convective boundary conditions", Comp. Nonlinear Sci. Numer. Simul., 17, pp. 4210-4223 (2012).

45. Khan, Z.H., Khan, W.A., Qasim, M., and Shah, I.A. "MHD stagnation point ferrofluid flow and heat transfer toward a stretching sheet", IEEE Transactions on Nanotechnology, 13(1), pp. 35-40 (2014).

46. Hamad, M. and Pop, I. "Scaling transformations for boundary layer flow near the stagnation-point on a heated permeable stretching surface in a porous medium saturated with a nanofluid and heat generation/absorption effects", Transp. Porous Med., 87, pp. 25-39 (2011).

47. Mustafa, M., Hayat, T., Pop, I., Asghar, S., and Obaidat, S. "Stagnation-point flow of a nanofluid towards a stretching sheet", Int. J Heat Mass Transfer, 54, pp. 5588-5594 (2011).

48. Ibrahim, W., Shankar, B., and Nandeppanavar, M.M. "MHD stagnation point flow and heat transfer due to nanofluid towards a stretching sheet", Int. J. Heat Mass Transfer, 56(1), pp. 1-9 (2013).

49. Hakeem, A.A., Ganesh, N.V., and Ganga, B. "Heat transfer of non-Darcy MHD flow of nanofluid over a stretching/shrinking surface in a thermally stratified medium with second order slip model", Sci. Iran, Transactions F, Nanotechnology, 22(6), pp. 2766-2784 (2015).

50. Hakeem, A.A., Govindaraju, M., Ganga, B., and Kayalvizhi, M. "Second law analysis for radiative MHD slip flow of a nanofluid over a stretching sheet with nonuniform heat source effect", Sci. Iran, Transactions F, Nanotechnology, 23(3), pp. 1524-1538 (2016).

51. Akram, S. "Nanofluid effects on peristaltic transport of a fourth grade fluid in the occurrence of inclined magnetic field", Scientia Iran, Transactions F, Nanotechnology, 23(3), pp. 1502-1516 (2016). 
52. Ghaffarpasand, O. and Fazeli, D. "Numerical analysis of MHD mixed convection flow in a parallelogramic porous enclosure filled with nanofluid and in the presence of magnetic field induction", Sci. Iran, Transactions F, Nanotechnology, 25(3), pp. 1789-1807 (2018).

53. Golshokooh, S., Ramazani, S.A., and Hekmatzadeh, M. "Investigating the effect of hybrid silica nanoparticles-copolymer on increasing oil recovery in a three dimensional porous media", Sci. Iran, Transactions F, Nanotechnology, 24(6), pp. 3466-3475 (2017).

54. Buongiorno, J. "Convective transport in nanofluids", J. Heat Transfer, 128, pp. 240-250 (2006).

55. Buongiorno, J., Venerus, D., Prabhat, N., McKrell, T., and Townsend, J. "A benchmark study on the thermal conductivity of nanofluids", J. Appl. Phys., 106(9), p. 094312 (2009).

56. Majeed, A., Javed, T., Ghaffari, A., and Rashidi, M.M. "Analysis of heat transfer due to stretching cylinder with partial slip and prescribed heat flux: A Chebyshev spectral Newton iterative scheme", Alex. Eng. J., 54(4), pp. 1029-1036 (2015).

57. Ghaffari, A., Javed, T., and Labropulu, F. "Oblique stagnation point flow of a non-Newtonian nanofluid over stretching surface with radiation: a numerical study", Therm. Sci., 21(5), pp. 2139-2153 (2017).

58. Mahmood, A., Chen, B., and Ghaffari, A. "Hydromagnetic Hiemenz flow of micropolar fluid over a nonlinearly stretching/shrinking sheet: Dual solutions by using Chebyshev spectral Newton iterative scheme", $J$. Magn. Magn. Mater., 416, pp. 329-334 (2016).

59. Uddin, M.J., Khan, W.A., and Ismail, A.I. "MHD forced convective laminar boundary layer flow of convectively heated moving vertical plate with radiation and transpiration effect", Plos One, 8(5), p. 62664 (2013).
60. Bianco, V., Manca, O., and Vafai, K., Heat Transfer Enhancement with Nanofluids, CRC Press, London, New York (2015).

61. Yih, K.A. "MHD forced convection flow adjacent to a non-isothermal wedge", Int. Commun. Heat Mass Transfer, 26(6), pp. 819-827 (1999).

62. Cebeci, T. and Bradshaw, P., Physical and Computational Aspects of Convective Heat Transfer, Springer, New York (1988).

\section{Biographies}

Muhammad Saleem Iqbal is an Assistant Professor in the Department of Mathematics at the Islamabad College for Boys G-6/3, Pakistan. He received a $\mathrm{PhD}$ degree from the Department of Mathematics and Statistics, International Islamic University Islamabad. His research interest is in the fields of heat and mass transfer, mathematical modelling, nanofluid dynamics, and entropy generation analysis of the similar and nonsimilar flows.

Abuzar Ghaffari obtained his $\mathrm{PhD}$ degree from the Department of Mathematics, International Islamic University, Islamabad, Pakistan. His research area is computational fluid dynamics. He is currently working as Assistant Professor at University of Education Attock Campus, Pakistan.

Irfan Mustafa received his $\mathrm{PhD}$ degree from the Department of Mathematics, International Islamic University Islamabad, Pakistan. His research area is computational fluid dynamics. He is currently working as a lecturer at Allama Iqbal University, Islamabad, Pakistan. 